\title{
Simulating naturalistic demands for speeded judgment
}

\author{
HAROLD H. GREENE, DAVID A. WASHBURN, and FERNANDO A. GONZALEZ \\ Morris Brown College, Atlanta, Georgia
}

\begin{abstract}
Responses to speeded judgment (e.g., "shoot/don't shoot") situations in naturalistic environments are influenced by many variables and psychological processes. We describe a custom-built simulator with head- and eye-tracking capabilities for basic and applied research on speeded decision making. The system allows the user to project life-sized, time-coded, videotaped, or computer-animated scenarios on a screen to collect reaction-time, shot-accuracy, and eye-movement data. An initial experiment is also reported to demonstrate the utility of the system for studying factors that affect speeded judgment under naturalistic situations.
\end{abstract}

Laboratory experiments on speeded judgment are common. From the earliest experiments in psychology to the present day, scientists have examined the effects of stimulus, response, and subject variables on the latency and accuracy of rapid decision making. Laboratory conditions have facilitated the manipulation of these variables, and the contemporary infusion of computer technology makes this research even more effective, efficient, and economical.

In contrast, it is difficult to examine speeded judgment under naturalistic conditions. Seldom do laboratory paradigms or findings generalize directly to time-critical decision-making environments such as driving a car, flying a plane, playing a sport, or responding to a shoot/ don't shoot (SDS) situation. These naturalistic decisions have been studied (see, e.g., studies of SDS judgments by Brown, 1984, and Holzworth \& Pipping, 1985), but typically with questionnaire or error-analysis methods unlike the chronometric procedures from the laboratory.

In the present paper, we describe a customized research tool for studying speeded judgment under conditions that simulate naturalistic environments. The basic process we study is SDS decision making, but the apparatus is equally appropriate for examination of other speeded and nonspeeded judgments.

\section{APPARATUS}

The central unit of our apparatus is a custom-made firearms training simulator. Seymour, Stahl, Swann, and Ross (1995) have demonstrated that the performance of police officers in response to videotaped SDS scenarios in a

This research was supported by Army Research Office Grant DAAL03-92-G-0382 to Morris Brown College Center of Excellence for Research on Training and by Grant N00014-95-1-1 100 from the Office of Naval Research. Reprint requests may be addressed to $\mathrm{H}$. $\mathrm{H}$. Greene, Center of Excellence for Research on Training, Morris Brown College, 643 Martin Luther King, Jr., Dr., N.W., Atlanta, GA 30314 (e-mail: harold@cert.atlanta.com). simulator and in response to actual, naturalistic events is comparable. Thus, the simulator approach would appear to provide a faithful way of producing naturalistic behavior in the laboratory. However, commercially available simulators are ill-suited for research purposes. They do not permit control and manipulation of stimulus variables (identity and timing of events in the scenario) and have to be modified to collect research data (see Seymour, Stahl, Levine, Ingram, \& Smith, 1994). They are ideal for training, but require substantial effort and cost to produce additional scenarios that instate specific experimental manipulations and controls. In contrast, the simulator we describe permits the use of any videotape, which can be recorded with experiment-specific content. This simulator is also compatible with computer-animated graphics scenarios of any length or content-popular computer games, artificial laboratory stimuli, text, or other images.

A Proxima (San Diego, CA) projector is used to display these taped or computer-generated images on a $3.25-\mathrm{m}$-wide $\times 2.2-\mathrm{m}$-high cinema screen. The screen is located $7 \mathrm{~m}$ from the projector, in a laboratory space in which other light or reflective sources are masked with black curtains. The simulator is illuminated by light from the projector reflected off the cinema screen. For the present demonstration, illumination around the participants averaged $0.053 \mathrm{~lm} / \mathrm{m}^{2}$. This level of illumination allows for good visibility of the projected images and the laser response device at arm's length. Two Pentium computers control this hardware. One controls the JVC BR-S500U (Fairfield, NJ) professional video cassette player to permit frame-specific indexing of all scenarios. The other computer generates computer-graphic displays. Both computers receive information from a Matrox (Dorval, PQ) digital frame-grabber via a camera, located atop the projector. The frame-grabber is used to register automatically any screen-directed response with a laser response device. Presently, a $9 \times 12.75 \mathrm{~cm}$ hand-held laser pointer and a $9-\mathrm{mm}$ handgun modified to shoot a laser beam are in use in the laboratory. 
The system automatically records numerous data. In addition to time-marked stimulus events, the computers record the time and coordinates of each response with the laser pointer (or other device such as a joystick, mouse, or keyboard). Additionally, eye-movement and pupil-dilation data are collected with an Iscan (Burlington, MA) RK-426pc head-mounted eye tracker. The eye tracker consists of two small cameras attached to a headband. A scene camera records everything that is in front of an observer's eyes (i.e., field of view). The other camera is focused on an ocular pupil and records eye movements and pupil size. Once calibrated, the RK-426pc will automatically calculate point of regard within the field of view and produce a cursor overlay onto the images from the scene camera. The output to the experimenter's monitor is the observer's field of view, with a cursor indicating what the observer is looking at within this field. For data analysis, the output can be recorded on videotape. In addition, digital data (the time-stamped coordinates of visual gaze and the size of the pupil) are recorded by the computer. The entire head-mounted Iscan unit weighs about $8 \mathrm{oz}$ and communicates with a Pentium computer either by $4.5-\mathrm{m}$ tether or with a telemetry system. In either case, the participant is free to move about during testing. The system typically provides point of regard within $1^{\circ}$ of accuracy over a $\pm 20^{\circ}$ range of pupil movement.

A TROTTER 685 (Medway, MA) wide-bed programmable treadmill and a BIOPAC (Goleta, CA) psychophysiological monitoring system are also available in the laboratory for use with the simulator. The treadmill permits the generation of active physical states (e.g., fatigue) that might occur in the environment. The psychophysiological monitoring system provides such measures as heart rate, blood pressure, body temperature, EEG, EMG, and skin resistance. These measures are a means of validating the realism of the simulator to the degree that participants manifest physiological responses appropriate for the scenarios.

\section{EXPERIMENT}

An experiment was conducted to illustrate the use of this apparatus and to investigate the generalization of findings from typical laboratory experiments. From lab- oratory findings, it is known that the time required to identify a target increases with increases in target complexity and with increases in number of distracting stimuli in the search task. However, salient targets can "pop out" and be quickly identified (e.g., Treisman \& Gelade, 1980). Laboratory findings have also shown that the visual system sufficiently acts as a Fourier analyzer (see Weisstein \& Harris, 1980, for a discussion) and simple reaction times (RTs) to higher spatial frequency information in a display are typically slower than those to lower spatial frequency information (e.g., Breitmeyer, 1975). Finally, movement in the visual scene will elicit an involuntary shift of attention (e.g., Breitmeyer \& Ganz, 1976), and attention can be voluntarily directed as a function of expectation or intention (Yarbus, 1967), all of which can affect RT to a target. In light of these typical laboratory findings, what should be expected for the following two questions? How will participants respond to a target (i.e., person with a firearm) if the target subtends a large versus a small visual angle? What is the effect on performance as participants become more familiar with the task? For the first question, we anticipated that response latency would be longer for the smallvisual-angle condition, given that response to higher spatial frequency information is typically slower than response to lower spatial frequency information. Contrary to this, one might also argue that the threatening biological motion in both visual angle conditions or pop out of the firearm might minimize this effect. For the second question, we predicted that performance would benefit from exposure to more scenarios, but one could also expect this short-term exposure to provide invalid cues regarding the location of the threat.

\begin{abstract}
Method
Twenty-one right-handed male students at the Atlanta University Center participated in the demonstration in exchange for payment. Their ages were between 18 and 36 years. No one had participated in a real-life SDS situation or had had any police experience. Each participant was tested on a series of 12 videotaped scenarios (see Table 1) using the simulator described above. For the present paper, only responses to the "visual angle" parking-lot scenarios will be described. The questions of concern were the difference in performance when the threat stimulus (the shooter) subtended a small or a large visual angle and whether performance in the small-visualangle condition benefited from short-term practice with the task. In
\end{abstract}

Table 1

Description of the Scenarios Currently Available

\begin{tabular}{lll}
\hline \multicolumn{1}{c|}{ Scenario } & \multicolumn{1}{c}{ Brief Description } & \multicolumn{1}{c}{ Variables Manipulated } \\
\hline $\begin{array}{l}\text { Alley } \\
\text { (4 scenarios) }\end{array}$ & $\begin{array}{l}\text { The participant sees an alley with a crowd of } 4 \text { to } 8 \text { people. Suddenly, the people } \\
\text { disperse to reveal a man or a woman, located in the middle of the group, aiming } \\
\text { and firing a weapon. }\end{array}$ & $\begin{array}{l}\text { a. Search set size (4 vs. 8) } \\
\text { b. Sex of shooter }\end{array}$ \\
$\begin{array}{l}\text { Apartment } \\
\text { (4 scenarios) }\end{array}$ & $\begin{array}{l}\text { An unarmed woman stands at the end of a hallway, screaming for help. A series } \\
\text { of three individuals, each requiring a shoot/don't-shoot response, emerge from } \\
\text { a door on either side of the hall. }\end{array}$ & $\begin{array}{l}\text { a. Armed vs. unarmed suspects } \\
\text { b. Ambient lighting conditions } \\
\text { c. With vs. without distraction } \\
\text { d. Sex of shooter }\end{array}$ \\
$\begin{array}{l}\text { Parking lot } \\
\text { (4 scenarios) }\end{array}$ & $\begin{array}{l}\text { An argument takes place in front of a row of cars. One unarmed individual } \\
\text { passes from right to left, another from left to right. An armed man (i.e., the } \\
\text { shooter) appears from between cars and fires his weapon. }\end{array}$ & $\begin{array}{l}\text { a. Visual angle of shooter } \\
\text { (2 scenarios) }\end{array}$ \\
\end{tabular}


the small-visual-angle condition, the shooter was $2^{\circ}$ wide and $6^{\circ}$ high, and the length of the firearm was $0.5^{\circ}$ before it was raised to shoot at the participant. In the large-visual-angle condition, the shooter was $3^{\circ}$ wide and $8^{\circ}$ high, and the length of the firearm was $1^{\circ}$ before it was raised to shoot at the participant. Participants were told to respond swiftly, whenever appropriate, by shooting at the torso of the perpetrator.

Following two scenarios, the individuals in one group (Group 1) saw the parking lot scenario in which the shooter subtended a large visual angle and individuals in the other group (Group 2) saw the scenario in which the shooter subtended a small visual angle. This between-group design permitted comparison of performance in the two visual-angle conditions. The locations of the shooter in the visual-angle conditions differed by about $5^{\circ}$.

To investigate the effect of practice on performance, we compared the performances of Groups 1 and 2 in the small-visual-angle condition. Group 1 (after having been presented with the largevisual-angle condition as scenario No. 3) performed in the smallvisual-angle condition as scenario No. 7. All intervening scenarios (Nos. 4-6) were selected from the other available scenarios and may or may not have required a "shoot" response. The data for Group 2 were those collected earlier in their encounter with the smallvisual-angle condition (i.e., scenario No. 3 for this group).

To ensure that observers were similarly sensitive to the visual stimuli in the videotaped scenarios on the screen, each participant's binocular contrast sensitivity was measured via the Pelli-Robson letter chart (Pelli, Robson, \& Wilkins, 1988) at the end of each experimental session. Weber contrast of letter triplets on the chart decreases from left to right in $0.15 \mathrm{log}$ units. From the participants position, each letter subtended a visual angle of $2.29^{\circ}$ and the smallest angle of resolution in the chart (i.e., the gap in the letter C) was $0.6^{\circ}$.

\section{Results}

With regard to the first comparison, mean shoot RT, measured from the appearance of the on-screen firearm was $566 \mathrm{msec}(S E=111)$ in the large- and $709 \mathrm{msec}$ $(S E=70)$ in the small-visual-angle condition, suggesting a trend in the anticipated direction. However, the difference was not statistically significant $[t(19)=1.11$, $p>$.05].

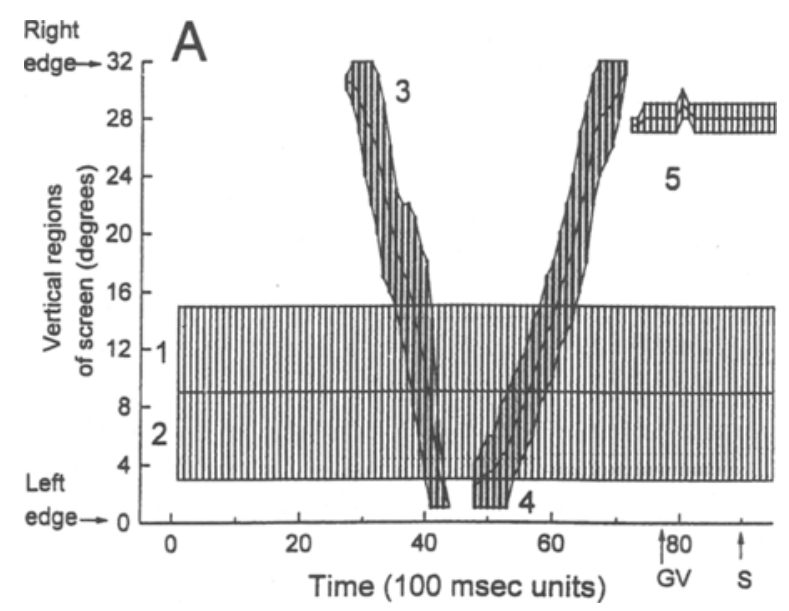

A significant difference was observed in performance on the small-visual-angle condition as a function of exposure to scenarios. Participants who encountered the small-visual-angle condition as scenario No. 7 responded reliably faster than those who viewed this condition early in the session as scenario No. $3[327 \mathrm{msec}(S E=84)$ vs. $709 \operatorname{msec}(S E=70)$, respectively; $t(19)=3.52, p<.05]$. This difference was not attributable to differential visual sensitivity to the on-screen visual stimuli, because there was no difference in the mean Pelli-Robson contrast sensitivities of the two groups [1.81 $(S E=.06)$ vs. 1.87 $(S E=.03) \log$ units; $t(19)=0.84, p>.05]$. Neither did the difference in RT appear to stem from the additional practice of the motor response that one group received (i.e., responding to 6 vs. 2 prior scenarios of any kind). For instance, although response latency was affected, there was no difference between the two groups in response accuracy. The distances between the "shot" of light emitted from the response device and the midtorso of the armed man were 100 pixels $(S E=15)$ in the less practiced and 92 pixels $(S E=12)$ in the more practiced group $[t(19)=-0.39, p>.05]$. It seems likely that cognitive factors in the form of facilitated recognition improved search strategy, or that a shift in signal-detection parameters accounted for the improved performance as a function of experience.

This interpretation is strengthened by analysis of eye movements during the scenarios. The events of concern in the scenarios occurred in the horizontal dimension (e.g., a man walking from right to left). As a result, visual search behavior was defined as the average horizontal point of regard within each $100-\mathrm{msec}$ period. Thus, a "gaze point" reflected fixations and small eye movements made within a given $100-$ msec period. For the purpose of comparing horizontal gaze points with on-screen events, the cinema screen was divided into 32 vertical regions,

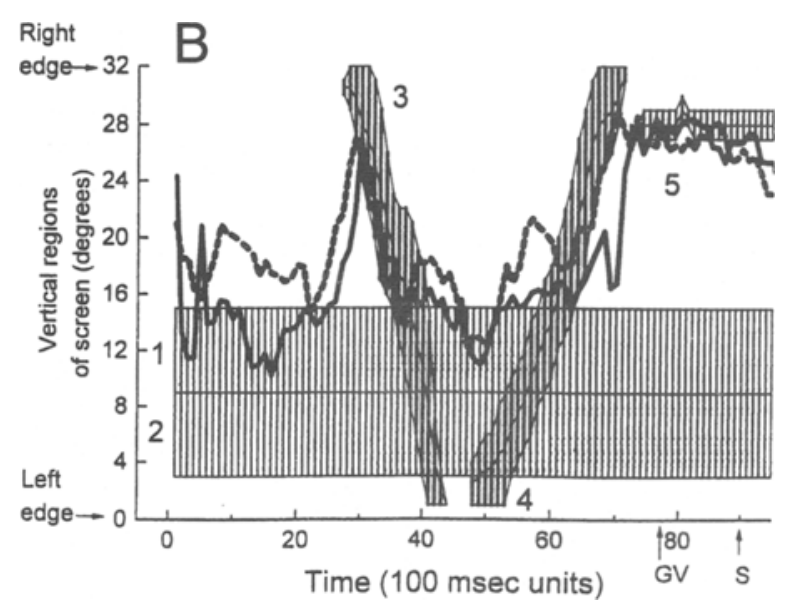

Figure 1. (A) A screen region-by-time representation of the parking lot scenario. The abscissa depicts elapsed time, and the ordinate depicts vertical divisions of the screen into 32 regions. An argument between a male (1) and female (2) occurred from scenario start to finish. At $2.7 \mathrm{sec}$ into the scenario, a male passerby (3) walked from right to left. At 4.7 sec, another male passerby (4) walked from left to right. The shooter (5) appeared $7 \mathrm{sec}$ into the scenario, $19^{\circ}$ from the argument. A gun became visible (GV) at $7.8 \mathrm{sec}$ and shot (S) was fired at 9 sec into the scenario. (B) Mean gaze data in 100-msec time periods for 11 participants who saw this scenario as scenario No. 3 (solid line) and for 10 participants who saw this scenario after responding to six other scenarios (dashed line). 
each subtending a visual angle of $1^{\circ}$. Figure $1 \mathrm{~A}$ depicts events in the parking lot scenario, and Figure 1B depicts participants' visual tracking of the events in the smallangle-condition parking lot scenario. These data illustrate a novel procedure for representing and analyzing visualtracking behavior in a dynamic visual scene.

Compare the solid-line with the dashed-line pattern of gaze in Figure 1B. The dashed line represents the mean gaze of participants in Group 1. The solid line represents the mean gaze of participants in Group 2, who had had less practice with the task and responded significantly more slowly than their counterparts in Group 1. Figure $1 \mathrm{~B}$ shows that individuals in both groups focused on the argument just left of midscreen and tracked the movement of figures across the screen. Participants in Group 1 (dashed line) spent less time than those in Group 2 (solid line) in gazing at the relatively stationary argument in the scenario. A $t$ test confirmed a lower mean gaze time in the argument area for Group 1, whose participants had had more scenario experience $[2.1 \mathrm{sec}$ $(S E=0.2)$ vs. $2.9 \mathrm{sec}(S E=0.3) ; t(19)=-2.36, p<$ $.05]$. Thus, pattern of visual search was affected by prior information.

\section{DISCUSSION}

Participants rapidly identified and responded to a threat stimulus regardless of its subtended visual angle. It is likely that movement by the armed man and the salience of his firearm elicited shifts of attention that minimized any visual angle (and spatial frequency) effects. Exposure to more scenarios did significantly facilitate these time-critical judgments. This facilitation appears to reflect changes in expectation and search strategy rather than a perceptual or motor artifact. Analysis of eye movements using a novel technique also revealed alterations in visual search behavior as a result of experience with other scenarios.

Of course, these findings follow quite reasonably from the existing literature. It is important to note, however, that the opposite effects could also have been predicted. Therefore, it is essential to identify the conditions under which certain laboratory findings generalize to real-life (or simulated) judgments and under which they do not. Under such complex and naturalistic conditions, numerous variables may vie for influence over speeded judgments, and it may be impossible to anticipate whether performance will be facilitated, inhibited, or unaffected. The present experiment demonstrates the usefulness of the simulator described here for applying laboratory techniques and results to naturalistic decision-making contexts.

\section{REFERENCES}

BreitMeyer, B. G. (1975). Simple reaction time as a measure of the temporal response properties of transient and sustained channels. $V i-$ sion Research, 15, 1411-1412.

BREITMEYER, B. G., \& GANZ, L. (1976). Implications of sustained and transient channels for theories of visual pattern masking, saccadic suppression, and information processing. Psychological Review, 83, 1.36.

Brown, M. F. (1984). Use of deadly force by patrol officers: Training implications. Journal of Police Science \& Administration, 12, 133-140.

Holzworth, J. R., \& PipPING, C. B. (1985). Drawing a weapon: An analysis of police judgments. Journal of Police Science \& Administration, 13, 185-194.

Pelli, D. G., Robson, J. G., \& Wilkins, A. J. (1988). Designing a new letter chart for measuring contrast sensitivity. Clinical Vision Sciences, 2, 187-199.

Seymour, G. O., Stahl, J. M., Levine, S. L., Ingram, J. L., \& Smith, R. F. (1994). Modifying law enforcement training simulators for use in basic research. Behavior Research Methods, Instruments, \& Computers, 26, 266-268.

Seymour, G. O., Stahl, J. M., Swann, G. B., \& Ross, D. (1995). Use of a computer-controlled firearms training simulator in perception response time experiments. Behavior Research Methods, Instruments, \& Computers, 27, 148-151.

Treisman, A., \& Gelade, G. (1980). A feature-integration theory of attention. Cognitive Psychology, 12, 97-136.

WEISSTEIN, N., \& HARRIS, C. S. (1980). Masking and unmasking of distributed representations in the visual system. In C. S. Harris (Ed.), Visual coding and adaptability (pp. 317-364). Hillsdale, NJ: Erlbaum.

Yarbus, A. L. (1967). Eye movements and vision. New York: Plenum.

(Manuscript received October 1, 1996; revision accepted for publication February 6, 1997.) 